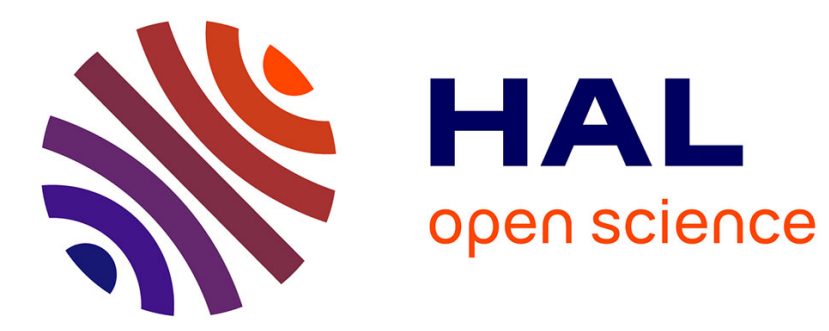

\title{
mettre en ordre les activités d'information, nouvelle forme de rationalisation organisationnelle \\ Brigitte Guyot
}

\section{To cite this version:}

Brigitte Guyot. mettre en ordre les activités d'information, nouvelle forme de rationalisation organisationnelle. Les Enjeux de l'information et de la communication, 2002, 2002. sic_00000355

\section{HAL Id: sic_00000355 \\ https://archivesic.ccsd.cnrs.fr/sic_00000355}

Submitted on 25 Jan 2003

HAL is a multi-disciplinary open access archive for the deposit and dissemination of scientific research documents, whether they are published or not. The documents may come from teaching and research institutions in France or abroad, or from public or private research centers.
L'archive ouverte pluridisciplinaire HAL, est destinée au dépôt et à la diffusion de documents scientifiques de niveau recherche, publiés ou non, émanant des établissements d'enseignement et de recherche français ou étrangers, des laboratoires publics ou privés. 
Mettre en ordre les activités d'information, nouvelle forme de rationalisation organisationnelle.

\author{
Brigitte Guyot, guyot@cnam.fr \\ Septembre 2002
}

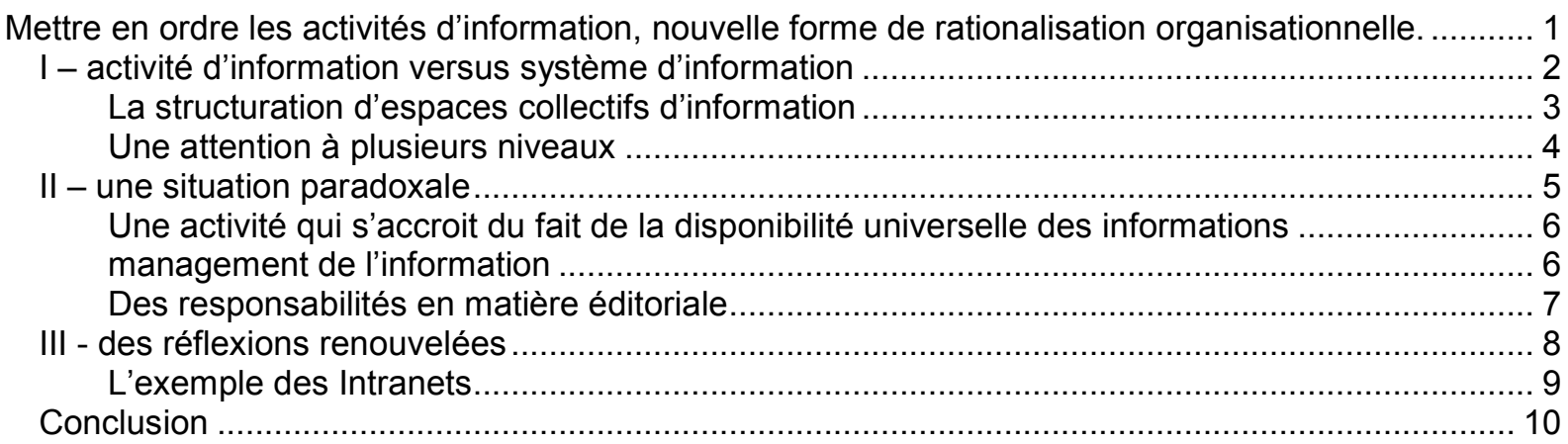

Pendant longtemps, la littérature française en information-communication abordait la question de l'information en la déclinant selon deux modes principaux, celui de la presse ou alors celui de la documentation. Cette position est réductrice, parce qu'elle fait l'impasse sur la complexité de ce qui est en jeu à propos de l'information, surtout lorsqu'il s'agit de l'information dans le cadre du travail. Partir alors de ce nous appelons activité d'information permet d'une part de souligner combien sont multiples les aspects engagés et d'autre part de mieux comprendre une partie des évolutions actuelles des organisations puisque cela ne sépare pas les aspects organisationnels, sociaux, relationnels des aspects proprement techniques ou linguistiques.

Lorsqu'elle est individuelle, cette activité a toutes les apparences du banal dans la mesure où chacun d'entre nous en fait l'expérience quotidiennement. La question est déjà moins banale quand il s'agit d'organiser la quête ou la transmission d'informations à l'intention d'autres personnes, ce qui revient à la considérer comme une activité de service. Les choses se compliquent encore lorsque ses modes d'organisation font l'objet d'une rationalisation, entendue comme une mise en ordre, d'autant plus que les technologies d'information focalisent le débat sur l'instrumentalisation de ce qui a longtemps été considéré comme une affaire de gestion et beaucoup moins comme une affaire de connaissance ou de relations de travail.

On pourrait s'interroger sur le manque d'écho des contributions des sciences de l'information tant à l'intérieur qu'à l'extérieur de la discipline. Hubert Fondin (2001) a avancé une première explication en relevant le poids prédominant de l'approche techniciste (dans laquelle l'information est entendue comme une donnée à formater pour effectuer des traitements ou une transmission) au détriment d'une approche sociologique. Pourtant, depuis une dizaine d'années, des études, principalement des thèses, ont abordé indirectement la question par le biais d'une sociologie des technologies (Lépine (2000), Martin (1997), Vacher (1997)) en s'attachant à relever les aspects organisationnels impliqués par la mise en place de technologies d'information ${ }^{1}$. Elles sont venues opportunément développer tout un champ de recherches dans ce qu'il est convenu d'intituler communication organisationnelle, complétant ainsi les apports d'autres disciplines qui ont plus ou moins directement abordé la question de l'information. Nous n'évoquerons ici que les sciences de gestion et la sociologie du travail qui ont alimenté les deux dimensions qui nous semblent importantes pour les recherches sur les questions informationnelles : la dimension managériale et la dimension des pratiques.

Considérer ensemble le plan de l'activité d'information et celui de sa mise en système permet de définir l'information non seulement comme une ressource mais comme un liant social et organisationnel, ce qui lie alors étroitement information et communication.

Pour nous, les mouvements observables actuellement sont liés à une tentative pour rationaliser non seulement les modes de gestion de l'information mais aussi les pratiques en la matière. Dans l'organisation rationnelle, qui attribue à l'individu une place en lui demandant d'être efficace, et lui alloue les moyens pour parvenir aux objectifs qui lui sont fixés, il y a une rationalisation qui s'exerce au plan collectif, par exemple pour gérer les ressources informationnelles, mais aussi dans les façons 
même de travailler et surtout de travailler ensemble, ce qui relève d'une exigence de rationalité intellectuelle.

La question de l'acteur est donc centrale parce qu'on ne peut pas réfléchir sur l'information sans lui associer le pôle de l'individu et le pôle de l'action. Selon les situations, l'acteur apparaît tantôt comme rationnel, prenant des décisions à l'issue d'un processus linéaire ; tantôt comme acteur social, engagé dans des stratégies de tous ordres, faisant preuve d'autonomie et d'initiatives, utilisant l'information selon le problème concret qui l'occupe ; parfois aussi comme acteur collectif, groupe de travail ou organisation tout entière, qui met en œuvre ou subit une politique d'information. En la matière, les pressions d'ordre cognitif et organisationnel sont nombreuses, et l'étude des réactions et stratégies permet d'analyser le niveau plus large des réorganisations en cours.

Ce mouvement transforme, enfin, une ingéniérie d'information encore trop souvent réduite à une informatisation peu soucieuse de l'organisation des contenus et de leurs modes de circulation. C'est parce qu'il y a une intégration progressive de ces aspects qu'on peut parler de management de l'information.

Les réflexions qui suivent résultent de recherches conduites depuis plusieurs années auprès de personnels cadres de services fonctionnels : plus d'une centaine d'entretiens ont été menés dans le secteur industriel et tertiaire à propos de leur activité de travail et de leur activité d'information, ainsi que de leur utilisation d'outils et de produits ; nous avons aussi suivi les transformations des modes de gestion d'information dans les entreprises et observé des pratiques informationnelles concrètes (utilisation d'un poste pour faire une recherche d'information par exemple).

\section{I- activité d'information versus système d'information}

Vouloir étudier la rationalisation de cette activité oblige à revenir brièvement sur les recherches sur l'activité de travail et de système d'information, en les resituant dans un contexte en évolution : la façon dont est appréhendée la question de l'information dans les entreprises est liée au contexte organisationnel et aux conditions de travail à distance, et bien sûr au développement des techniques communicantes.

La question de l'information a été très tôt abordée par les gestionnaires, qui l'ont tout d'abord considérée comme un facteur de stratégie pour l'entreprise, totalement liée à l'activité de décision et à l'activité de gestion, les préoccupations de l'encadrement étant en effet d'organiser le travail, de veiller à la bonne marche de l'entreprise en contrôlant ses activités et en anticipant ses choix stratégiques. La mise à plat du processus décisionnel a permis d'identifier des phases censées, par leur enchaînement séquentiel, se conclure par une décision éclairée, c'est-à-dire rationnelle. (Simon, 1955). A une bonne information correspondrait une bonne décision, construite automatiquement à partir de l'analyse de données hétérogènes qui se transforment en information lorsqu'elle font sens pour l'acteur par rapport à ses objectifs. Mais la surdétermination d'un processus logique, et d'une rationalité intrinsèque à la décision, focalisée sur le contrôle de l'incertitude, ont entraîné un modèle mécaniste progressivement remis en cause par plusieurs facteurs: tout d'abord, la notion de rationalité limitée de l'acteur reconnaît le fait que de nombreuses décisions ne sont pas prises en toute connaissance de cause, et qu'elles ne s'appuient pas uniquement sur des données structurées et validées, ainsi que le souhaiteraient les partisans des systèmes informatisés d'aide à la décision (SIAD). Ensuite, l'accent mis sur la dimension collective de la décision la montre comme une activité négociée. March soulignait, quant à lui, combien les jeux stratégiques autour de l'information viennent la connoter et en faire un outil de pouvoir, de manipulation, un alibi ou une arme (March, 1987).

Bruno Latour formulait la même chose un peu différemment, lorsqu'il montrait combien l'information résulte d'une succession d'opérations d'abstraction progressive par rapport au monde réel. Le prélèvement d'un échantillon de terre, son transport en des lieux d'analyse et sa transformation en éléments significatifs mis sur des supports susceptibles d'être traités, agrégés, comparés, participe d'une certaine façon de la décision, en tant qu'elle constitue une autre face de l'interprétation du monde environnant (Latour, 1996).

Les années récentes ont vu l'information devenir un facteur de compétitivité dans un monde devenu concurrentiel à l'échelon mondial. Elle entre ainsi dans le capital immatériel de l'entreprise, au même titre que les logiciels, la formation, le portefeuille d'innovation, ou les ressources humaines, encore 
qu'elle figure rarement sous cette appellation. (revue française de gestion 2000). Richesse dont l'évaluation économique reste problématique tant que les critères classiques de productivité et de retour sur investissement continueront de lui être appliqués.

En interprétant de façon large les analyses gestionnaires de la relation entre l'activité et l'information, celle-ci apparaît comme venant doubler l'activité elle-même, à la fois image et preuve de son existence. Elle se superpose au déroulement de l'activité, qu'elle rend visible et traitable. Toute activité peut se traduire par une donnée, que ce soit un chiffre d'affaires, un coût de fonctionnement, une consommation de moyens ou un résultat. Cette information de gestion procure une vision surplombante utile au contrôle et à l'allocation des ressources. Mais on sait bien que les chiffres ne traduisent qu'une certaine partie de cette réalité.

L'analyse de l'activité la fait apparaître fortement demandeuse d'information, mais aussi encadrée par toutes sortes de directives et ou textes qui la limitent tout en la soutenant. Les travaux réalisés sur les règles dont sont dotés, ou se dotent, les acteurs (Reynaud, 1993) et ceux sur les conventions qui permettent aux acteurs de travailler ensemble, ont permis d'approfondir la façon dont ceux-ci jouent avec ces cadres, s'y conforment ou les modifient (Réseaux, 1993). Ils ont montré l'intense activité de négociation, de communication, de production ou d'agencement de règles qui s'instaure autour de l'activité pour la réguler. C'est reconnaître le fait que toute activité, pour se réaliser et en se réalisant, utilise et produit de l'information. L'acteur a besoin de savoir ce que font ceux qui travaillent avec lui afin de se coordonner avec eux.

De leur côté, les sociologues du travail ont insisté sur la part d'autonomie que possède l'acteur, mesurable à la façon dont il choisit les moyens mobilisés pour effectuer son travail (Terssac); conjointement, il mène une intense activité sociale pour construire une représentation partagée de l'activité à laquelle il participe afin de se coordonner avec ses collègues. Le terme d'information signale à la fois les moyens engagés, et les communications interpersonnelles qui contribuent à développer une inter-compréhension (Zarifian, 1996). Cela rejoint la question des activités langagières engagées dans la coopération et dans le travail d'équipe.

L'information apparaît alors fortement liée au monde mouvant où choses et signes s'entrecroisent en fonction des acteurs qui s'en saisissent ou la manipulent selon leurs intérêts. Nul n'en doute, à vrai dire, mais ce rappel permet d'insister sur le fait que, par exemple, l'information n'est pas un en-soi à regarder avec respect, attitude qui imprègne encore fortement notre culture de l'écrit et notre relation à la connaissance. La mise en banques de données et en outils tend à naturaliser, donc à passer sous silence, tous les traitements intermédiaires qui constituent autant de décisions et d'interprétations subies par l'information, et dont elle garde trace de façon invisible ou implicite pour celui qui s'en saisit.

II nous paraît donc plus juste de considérer l'information comme un produit social toujours ouvert, sans cesse ré-interprété par l'usage qui en est fait (Jeanneret, 2000), dans le but de clarifier les composants de l'intense dynamique sociale au sein de laquelle elle est produite et exploitée, donnant lieu, notamment, à des activités de collecte, de tri, d'évaluation, de traitement, de négociation, de mise en forme et de classement.

\section{La structuration d'espaces collectifs d'information}

La mise en système de ces flux multiples, qui concerne aussi bien l'information de fonctionnement (utile pour agir), l'information de gestion, l'information en provenance de l'extérieur (surveillance), que l'information produite en interne de façon individuelle (dossiers de travail), rend la question des systèmes d'information tout à fait centrale. Les sciences de gestion, l'ont travaillée en la pliant à leurs préoccupations, dans une perspective de modélisation. Malgré les connotations fortement techniques du terme, il offre une matrice féconde à condition d'être élargi et d'y intégrer les apports des réflexions sur les dispositifs, qui insistent, notamment, sur le système d'acteurs.

Le système d'information, tel que l'entendent gestionnaires et informaticiens, correspond au système général de gestion, ce qui marginalise d'emblée tous les autres sous-systèmes dévolus à d'autres activités, telles que la qualité, la veille concurrentielle ou les ressources humaines ou pose en tout cas la question des rapports qu'ils entretiennent entre eux. Centralité, transversalité, périphérie, autonomie, sont des question fort centrales pour réfléchir sur le sujet. Les réflexions systémiques ont 
fortement évolué depuis vingt ans, après s'être calées sur l'organisation hiérarchique pour expliquer la place d'un système d'information. Elles le situaient comme un intermédiaire entre les lieux de décision et les opérationnels, le système centralisant les informations du terrain destinées aux décideurs pour, en retour, les redistribuer sous forme de directives ; aujourd'hui il est considéré comme un pôle commun rassemblant et traitant tous types d'informations, irriguant l'entreprise et lui permettant de construire une représentation partageable par l'ensemble de ses membres (Le Moigne, 1977, Bartoli, 1996). Cependant, dans ces constructions, l'acteur reste un élément du système sans consistance concrète. II ne s'agit pas de nier l'intérêt de cette position mais de souligner son caractère inopérant pour analyser les niveaux locaux, que constituent par exemple un service ou un collectif de travail. Le modèle théorique cède la place au foisonnement social des habitudes de travail, des conflits d'intérêts, ce qui demande d'autres outils d'analyse.

Cela suppose alors de dépasser la vision globale et instrumentale (traiter des données) du système d'information, pour travailler sur le dispositif social, comprenant un certain type d'acteurs, les règles dont ils se dotent, des objets qui s'échangent ou circulent, éventuellement des outils qui traduisent les choix organisationnels.

Car dans une entreprise, le système général est composé (certains voudraient le voir les englober) de nombreux systèmes d'information qui n'impliquent pas les mêmes personnes ni les mêmes circuits mais qui s'interconnectent quelque part. Ainsi tout salarié émarge à plusieurs systèmes, celui des ressources humaines, de la communication, en plus de celui de son propre service. Un service, commercial par exemple, s'organise pour collecter, traiter et faire circuler aussi bien l'information de gestion, concernant par exemple les résultats de chacun, que les informations dont le personnel a besoin pour travailler et se tenir au courant des évolutions des produits et du marché (catalogues, dossiers concurrents). Dans ce qui apparaît comme un même système d'information local se constate l'existence de plusieurs niveaux, l'un, hiérarchique ou administratif pourrait-on dire, dévolu à des fins de suivi et de contrôle, qui cohabite avec un niveau exécutif ou opérationnel regroupant les moyens et les ressources d'information. Ils fonctionnent selon des impératifs, des temporalités et même des spatialités d'ordre différent: certains travaillent sur le passé, d'autres sur la prospective, certains concernant l'ensemble de l'entreprise ou au contraire en appui d'une fonction spécifique. Certains sont laissés à la libre disposition de chacun ou au contraire sont d'un accès réservé, d'autres peuvent être alimentés de façon impérative. La diversité de ces sous-systèmes d'information locaux, à la fois en relation avec le niveau global (par exemple par le biais des formulaires de gestion à rendre chaque mois) tout en étant totalement spécifiques, pose, pour celui qui veut les intégrer dans un système commun, des problèmes qui ne sont pas seulement techniques mais qui sont liés à la culture de chaque entité et à une politique générale en matière d'information.

\section{Une attention à plusieurs niveaux}

Ainsi, l'acteur (individuel ou collectif) développe une activité d'information plus ou moins forte : il se dote de règles pour en produire, pour en communiquer et en utiliser. II adopte une conduite raisonnée pour chercher celle qui l'aide à résoudre les problèmes concrets de son travail, en fonction du contexte organisationnel et de l'action envisagée ou entreprise. II endosse une responsabilité, dans la mesure où il a les moyens d'analyser son activité et de mesurer les conséquences de ses actes, d'en rendre compte et de prendre un certain nombre de décisions qui le concernent directement. L'information est donc toujours située, c'est-à-dire qu'elle est sans cesse passée au crible de la situation particulière de l'individu. Cela pose problème dès lors qu'on veut organiser un pôle de ressources commun puisqu'il va s'agir d'articuler ces systèmes personnels.

Il est donc tout à fait intéressant de coupler ce premier niveau individuel (local) avec un regard qui remonte successivement vers des niveaux plus larges et plus globaux. Cela permet d'articuler une focale gestionnaire, concentrée sur l'encadrement du travail, avec une focale sociologique, penchée sur les choses en train de se faire, les façons d'être ensemble, sans parler des aspects proprement cognitifs que nous n'aborderons pas ici. II semble que les sciences de l'information-communication, et plus particulièrement la communication organisationnelle, se situent à l'intersection de ces approches. Etudier le processus qui met en système oriente l'attention sur l'évolution des cadres de travail et des façons de travailler et de se coordonner. Cela suppose, sur le plan méthodologique, d'allier l'observation des acteurs avec l'étude des traces considérées comme révélatrices de leur activité, toutes deux venant compléter les discours qu'ils peuvent porter sur leur activité et sur les outils qui les entourent. 
Cette activité d'information s'inscrit toujours dans un système d'information plus large, système social avant tout. II peut être considéré comme un projet inscrit dans des stratégies et des contraintes de différents ordres et en interaction dynamique : communicationnel, dans les relations entre les acteurs et les objets qui circulent entre eux; organisationnel, avec les procédures de travail et de coopération, le système de règles, locales et en lien avec l'organisation, avec le mouvement de rationalisation progressive des processus de travail ; cognitif, dans les façons dont se forge et se modifie un système de représentations (en relation avec l'identité, le projet, la motivation); dans les façons de penser, d'interpréter ou de calculer en utilisant les technologies. Enfin, comme tout projet collectif situé et contextualisé, il pousse à réfléchir l'engagement des acteurs, sur la confiance, sur la négociation, sur les intérêts et le bénéfice qu'ils peuvent tirer du système.

Raisonner en termes d'interaction demande donc d'approfondir la façon dont un acteur se dote d'un système d'information personnel, constitué de tous les circuits interpersonnels d'informations plus ou moins formalisées et d'outils techniques qu'il utilise pour s'informe en adéquation avec sa situation de travail. L'activité d'information révèle une variété de tâches très concrètes, dans lesquelles l'acteur est tour à tour décideur (choisir quoi chercher et où), gestionnaire (de la matérialité des documents dont il s'entoure) et producteur d'information (pour lui et pour ses collaborateurs). Toutes ces aspects donnent la mesure de cette activité extrêmement personnelle, qui se trouve sans cesse reliée au plan collectif, par l'activation de circuits ou d'outils mis en place pour travailler ensemble.

\section{II - une situation paradoxale}

A partir du moment où nous soutenons que l'information reflète l'état de l'organisation, l'étude de l'activité d'information concrète combine une approche au plus près de l'acteur sans perdre de vue les le point de vue managérial, ce qui produit un matériau explicatif tout à fait intéressant. Ainsi, la mise en ordre progressive de cette activité se révèle paradoxale : les organisations cherchent à la rationaliser en proposant par exemple des ressources directement accessibles, ce qui responsabilise l'individu dans le choix de ses moyens d'information; mais en même temps, elles renforcent les mesures encadrant, régulant, voire limitant cette autonomie informationnelle.

Après la première vague d'informatisation des données de gestion, les possibilités d'interconnexion des outils et leur mise en réseau ont poussé les entreprises à homogénéïser leur plate-forme technique et à faire dialoguer des îlots auparavant dispersés. Mais cela ne résoud qu'une partie du problème dans la mesure où centraliser des informations sur un même support dépasse largement les aspects techniques de formatage ou de norme. Cela pose des problèmes organisationnels spécifiques selon le système social et le type d'information concerné. Ce sont dorénavant tous les acteurs, aussi bien les manageurs les informaticiens ou les utilisateurs, qui sont impliqués par la réalisation de systèmes d'information collectifs, que ce soit au sein de leur propre service, ou pour produire une information pour le réseau.

Une autre évolution sensible se rapporte à l'élargissement des informations dorénavant prises en compte : après les données de gestion puis les données factuelles, du genre carnets d'adresse ou listes de produits, ce sont maintenant les documents informels des personnes (documents de référence et dossiers de travail) qui sont structurés et centralisés sur un support électronique unique ; les échanges eux-mêmes sont dorénavant mis en mémoire. Dans une dernière étape, ce sont les savoir-faire et les expertises individuelles qui font l'objet d'un regroupement puis d'un traitement. Or sélectionner ce qui, dans les dossiers personnels avant un départ à la retraite, peut être ré-exploité par son remplaçant va au-delà d'une simple opération d'identification et de tri. II suppose une construction d'un usage anticipé, d'une projection sur le type de question que cette information servira à résoudre. Substituer une banque de données d'informations structurées au processus de transmission directe de savoir-faire par apprentissage dépasse la formalisation des connaissances. Cela touche directement à l'histoire et à l'identité de la personne. Certes, bureautique, banques de données, messageries, Intranets et autres groupware, font désormais partie de l'environnement de travail quotidien. Mais les présenter comme de simples outils équivaut à oublier qu'une donnée chiffrée, un article scientifique, un rapport d'activité, un dossier produit ou un rapport de surveillance, diffèrent dans leurs conditions de production, dans leur organisation et même dans leur exploitation. Le déficit actuel d'attention à ces questions a déjà commencé à pesre lourdement sur l'usage de ces systèmes. Ce n'est pas tant la distorsion entre les volontés affichées et la réalité qu'une absence d'adéquation avec les pratiques réelles. 
Une activité qui s'accroit du fait de la disponibilité universelle des informations

Le développement des réseaux internes illustre une partie des contradictions auxquelles l'entreprise est confrontée. Présenté comme un nouveau circuit venant simplifier les circuits d'informations antérieurs souvent invisibles car informels, l'Intranet se rajoute aux réseaux existants, tout en apportant une logique d'accès qui tend à se substituer à la logique classique de diffusion. Chacun a déjà expérimenté la différence qui existe entre trouver sur son bureau la dernière lettre du président et devoir aller la chercher sur le réseau. Ce passage à une démarche volontaire induit des changements qui jouent sur plusieurs plans : social, parce que les circuits personnels sont modifiés ; cognitif, parce qu'il faut nécessairement identifier au préalable le type d'information recherchée, pour qu'elle corresponde à ce qui est susceptible d'être trouvé. Documentaire, enfin, parce que naviguer dans des classements et moteurs de recherche suppose des logiques particulières encore fort hétérogènes. $\mathrm{Et}$ tout ceci pour un utilisateur final qui cherche, avant tout, à résoudre un problème concret, et non à naviguer dans un univers de plus en plus touffu et rendu opaque par la prolifération des ressources informationnelles à sa disposition.

C'est pourquoi la stratégie qui consiste à laisser ces nouveaux réservoirs à la discrétion de l'utilisateur amplifie leur activité d'information avec des effets en matière de gestion du temps, d'implication et de compétences encore largement sous-estimés. Tout se passe comme si ce qui correspond à une logique de l'offre et qui postule une égalité de tous devant l'information, procède d'une méconnaissance des pratiques réelles et des implications socio-cognitives concrètes. Certes, chacun se construit quelques chemins régulièrement empruntés, mais les remarques abondent sur le temps d'investissement nécessaire, les difficultés rencontrées, que ce soit à propos des sources ou de la validation de l'information. Or il ne faut jamais perdre de vue que l'activité d'information n'est jamais qu'une activité qui vient en appui du travail mais non l'activité principale.

Sans nier l'importance des premières initiatives pour centraliser des données auparavant dispersées, celles-ci conviennent avoir largement sous-estimé l'importance de ce que nous appelons les informations situées, c'est-à-dire celles qui font sens pour l'acteur immergé dans son propre contexte. Les entreprises commencent à percevoir que la mise à disposition du maximum d'informations ne rend pas celles-ci automatiquement pertinentes ni exploitables. Même si les ingénieurs ou chargés de mission reconnaissent l'utilité de centraliser toutes les informations internes produites ou détenues par chaque service, ils insistent sur les difficultés pour les retrouver, du fait de leur mauvaise identification, de la façon dont elles sont organisées, sans parler des limitations d'accès. Identifier, classer, mettre en catégories se fait au gré des politiques, à l'échelle des services ou selon les projets. Alors que les discours officiels insistent sur les bénéfices d'une centralisation pour développer une logique de partage, la situation sur le terrain montre une balkanisation venant les contredire. Les entreprises oscillent entre la protection et le partage de leurs informations, parlant de partage là où il n'y en réalité qu'une simple mise à disposition.

Mais comme souvent, les premières expériences sont porteuses d'évolution. Devant la prolifération des informations ainsi engrangées, les entreprises commencent à se préoccuper des procédures de sélection et de validation, de systèmes de catégorisation pour orienter l'exploitation des informations. Les concepteurs commencent à prendre en compte l'utilisateur en accordant une attention aux intérêts locaux, et en cherchant à avoir connaissance des pratiques réelles. Mais, lorsqu'on dit concepteur, de qui parle-t-on?

\section{management de l'information}

Ces questions participent de la constitution de ce que nous appelons un management de l'information. La mise en place de systèmes d'information, locaux ou transversaux, ainsi que leur gestion, leur animation, suivi et contrôle, relèvent bien des responsabilités managériales. Décider d'un Intranet pour son département, le faire vivre au quotidien ou veiller à son adaptation aux intérêts de chacun (à son usabilité, dirait Le Coadic, 1998) demande d'être attentif à plusieurs niveaux. Celui de la diversité, et donc de la spécificité des informations et de leur fonction, c'est-à-dire leur utilité dans le contexte spécifique du service. Les données non structurées, représentées par du texte intégral, notes de service ou rapports de mission, continuent de poser des difficultés aux informaticiens, qui ont longtemps géré des données factuelles structurées, c'est-à-dire organisées en champs, pour en faciliter le calcul et le transfert.

Mais l'intégration de ces informations quotidiennes produites en interne ou celles qui proviennent de l'extérieur et qu'internet rend disponibles sans intermédiaire, pose problème au management qui n'est 
jamais totalement entré dans ce type de réflexions. Vouloir mutualiser des ressources ou décider que ses collaborateurs partager des informations personnelles, exige des stratégies différentes qui sont du ressort du management de l'information, pour analyser l'existant définir les objectifs communs et négocier de nouvelles règles, pour faire reconnaître et entourer l'activité d'information à l'échelle du service. La notion de système d'information prend donc des dimensions très concrètes, explorant des aspects liés à la psychologie, aux rapports personnels, aux façons de travailler à plusieurs, développant une réflexion sur les contenus susceptibles d'être mis en commun.

Ce qui remarquable dans un tel management c'est qu'il n'est plus uniquement l'apanage des spécialistes ni même des manageurs en titre mais prend des formes distribuées. Dans un service, la rationalisation des procédures d'information et de communication peut échoir à des cadres pour qui ces questions sont nouvelles, mais un leader peut se déclarer intéressé et vouloir s'y investir... Certains professionnels de l'information développent des activités d'audit et de conseil pour promouvoir une politique et une culture de l'information, mais la tâche est éminemment à la fois très politique dont les aspects très concrets supposent une proximité quotidienne : animation d'équipe, opérations d'adhésion ou d'incitation, pouvant aller jusqu'à la mise en place de formules pour rétribuer ceux qui partagent leur information. Où se définit la légitimité pour assumer ces tâches ? Les formules actuelles sont contrastées, entre laisser-faire, mesures autoritaires ou recherche de consensus. La prise de conscience du rôle joué par l'information, la définition ou la réorganisation des rôles et des nouvelles responsabilités ne recouvrent plus nécessairement les positions hiérarchiques existantes. Si certains métiers se recomposent vers des fonctions plus techniques ou plus éditoriales, ce qui est le cas par exemple des documentalistes, nous avons surtout constaté la montée de fonctions composites, assurées en complément d'une activité principale.

Mais ce management de l'information est à peine identifié, stabilisé, que les entreprises se lancent dans un management qui concerne également les connaissances et non plus seulement les informations, ce qui constitue une étape encore plus complexe. Mettre en système un bien personnel considéré comme indissociable de la personne, engage des niveaux d'analyse dont, pour le moment, beaucoup d'entreprise semblent faire l'économie. Implanter une solution technique peut, au mieux, s'expliquer par une logique de rationalisation liée au départ de personnels expérimentés. Ces initiatives ne peuvent faire l'impasse d'une réflexion sur la façon dont fonctionne un collectif, sur le type de contributions individuelles pour construire un référentiel commun. Elles peuvent s'analyser, de façon positive, comme une forme de reconnaissance des richesses d'une personne ou, de façon négative, comme le souci d'utiliser au maximum ses ressources, s'aventurant ainsi dans ce qui touche de près à son intimité. Cela peut expliquer la prudence actuelle des entreprises qui s'en tiennent à l'organisation des informations détenues par leurs membres.

\section{Des responsabilités en matière éditoriale}

Tout ceci nous amène à réfléchir au rôle et à la place de l'écrit dans le travail, et à celle des technologies intellectuelles. Dès 1951 Wright dressait un tableau saisissant des bureaux administratifs confrontés au recueil et à la circulation des données provenant du terrain (Wright 1951). Cette mise en écrit s'est encore accélérée avec le développement de la démarche la qualité, qui a quitté la production pour gagner progressivement tous les services fonctionnels. Elle s'appuie sur une opération d'explicitation de ce qui restait encore pour une large part d'ordre informel, cette formalisation et normalisation des procédures de production donnant naissance à des documents de référence. Le mouvement général est général et aucun acteur n'est épargné.

Car la stabilisation de l'informel transforme les modes de communication, les façons de regarder son activité, d'en rendre compte ou d'intervenir sur celle des autres. Le document devient un support de coordination et non plus simplement un support de gestion ou de justification. En effet, la montée des relations à distance, les participations à des projets de coopération entre entités différentes, suppose des points d'accord et une mise à plat des relations qui passe par l'écrit et la codification.

L'acteur a toujours écrit pour lui-même, mais il y a une différence entre ce type d'écrit, forme première de sa réflexivité, et l'opération qui consiste à organiser ses écrits pour les rendre disponibles et compréhensibles par d'autres (Delcambre, 1996). En entreprise, chacun écrit dans des cahiers, notes, dossiers personnels ou fichiers d'adresses créés pour son usage personnel et qui sont la marque de son autonomie. Le passage à une publicisation suppose des règles, c'est-à-dire la construction d'un consensus, pour organiser l'accès, l'enrichissement et la circulation de ces documents. C'est en ce 
sens qu'on peut parler de fonction éditoriale, en l'entendant comme dépassant la simple mise en forme (normalisation des formats et du type de contenu, voire des termes employés). Elle définit aussi le statut du document, répartit les rôles et les responsabilités (d'auteur, de lecteur, de contributeur ou d'administrateur), assure sa traçabilité et son cycle de vie (mises à jour éventuelles, archivage) sans oublier les modalités de sa circulation (modes de consultation). Ces responsabilités ne sont pas du même ordre, et semblent, pour le moment, très diversement assurées

Considérer le document comme un objet intermédiaire qui inscrit et organise des actes personnels ou collectifs, contribuant à construire une représentation partagée, permet d'adopter ce que nous appelons l'approche par le document. Elle consiste à analyser celui-ci sous différents angles : comme un support d'inscription (de visualisation, d'explicitation, de distanciation) de l'activité, comme le montre l'organisation du bureau électronique personnel; lorsqu'il a un statut de document collectif, regarder comment il inscrit les différentes interventions des acteurs ${ }^{2}$, les règles qui définissent sa production et les interactions autour de lui. Cette approche articule les dimensions éditoriales, organisationnelles et sociales en partant d'un objet concret pour le rapporter aux dynamiques sociales, sémiotiques qui s'inscrivent en lui.

\section{III - des réflexions renouvelées}

Dès lors qu'elle s'implique dans l'activité d'information, directement liée à l'organisation du travail, et qu'elle intervient sur les supports de relation, l'entreprise est amenée à maîtriser le projet technicoorganisationnel et les implications d'ordre socio-cognitif. Celles-ci se révèlent beaucoup plus complexes du fait qu'elles touchent de près des personnes et des collectifs dans leurs activités et leurs relations de travail, d'autant plus que les pressions nouvelles viennent s'exercer sur eux, aussi bien en termes d'identité que de relations. L'existence d'une sphère électronique renouvelle la question de la relation entre individu et information, entre sphère privée et sphère collective, ce qui renvoie, pour une part à la question de l'intimité (privacy, disent les anglais (Doty, 2001)) liée à celle de l'identité.

Les spécialistes de l'identité lui attribuent trois dimensions : l'identité pour soi est définie par l'image qu'une personne se fait d'elle-même, l'identité pour autrui par ce qu'elle donne à voir aux autres (Barbier, Kaddouri, 1996), et l'identité attribuée (''image que les autres se font de lui). C'est cette dernière qui, au travers des réseaux, entre en conflit avec les deux autres. Au cours de ses navigations et correspondances, une personne laisse derrière elle des identifiants qui, s'ils sont capturés et traités statistiquement, révèlent son comportement et contribuent à établir une identité électronique qui correspond rarement avec l'identité telle que la vit la personne ou qu'elle la présente aux autres. De même, il y a une grande différence entre le fait de définir soi-même son profil d'intérêt pour recevoir des informations, ou être défini automatiquement par son appartenance à une fonction ou à un service, afin d'être destinataire d'informations sélectionnées sur ces seuls critères. Il y a là des formes d'intrusion insidieuses dans l'intimité, ce qui n'est pas sans effets sur les mouvements actuels qui mettent en machine des informations liées aux personnes..

L'intimité, c'est aussi la liberté de donner de l'information et la liberté de choisir ses relations, ce que chacun fait avec ses réseaux personnels. En entreprise, il y a une dialectique du secret ou de la révélation censée s'exercer au bénéfice d'intérêts collectif et un mouvement d'auto-régulation, entre garder pour soi et divulguer, décuplé par la mise en place de systèmes d'information mutualisés venant se juxtaposer à ceux que les personnes ont développés et qu'elles peuvent jalousement vouloir préserver. II est, de ce point de vue, tout à fait intéressant d'étudier comme se réalise la gestion de la confidentialité.

Dans le même temps, la sphère électronique met l'individu devant une logique grandissante de notoriété, sous forme d'incitation à produire de l'information ou à se présenter sur le réseau. Cette situation occasionne des pressions contradictoires, et il lui faut gérer sa mise en visibilité ou au contraire sa protection, dans une situation où les règles, aussi bien éditoriales de confidentialité que de bon usage, restent encore largement implicites et rarement négociées, tout en étant progressivement intériorisées. Devoir partager ses informations personnelles incite certains à ne plus rédiger les compte-rendus autrefois oraux, de peur d'en être dépossédés ou de se donner de nouvelles contraintes.

L'intimité, en tant que perception de soi, renvoie à la responsabilité, c'est-à-dire à la manière d'évaluer les conséquences de ses actes et d'en répondre. Ne pas valider une information, oublier de la mettre 
à jour, peut avoir de lourdes conséquences. Signer un document, c'est se déclarer prêt à rendre des comptes. Le principe administratif d'anonymat ne tient plus avec les possibilités d'identifier la source, et de suivre les contributions sur un même document. Quel est le statut du document collectif lorsqu'il est produit par ajouts successifs, comment se distribuent alors les responsabilités en matière d'information?

Cette gestion des relations d'information pousse à réfléchir de façon plus générale à la façon dont sont mis en concordance les intérêts personnels et l'intérêt général, aux rapports de cette sphère privée avec la sphère collective. Le partage des dossiers personnels sur un serveur commun réactive des questions comme la possession et la propriété. Par-delà le cas extrême d'un brevet, dans lequel l'auteur partage avec l'entreprise une partie de ses droits, le développement de chartes de bonne conduite en la matière, prouve que cette question commence seulement à être clarifiée.

Parmi les termes les plus couramment employés pour annoncer la création de systèmes collectifs, ceux de circulation, de partage et d'échanges d'information semblent des formules incantatoires qui font l'impasse sur les implications concrètes de leur application. Mutualiser une ressource signale un rapport double et simultané fondé sur l'égalité des deux parties, par répartition et distribution. La circulation suppose qu'au moins deux points sont en relation. Le partage insiste sur le processus et les conséquences d'une répartition, sans préjuger de l'égalité des parts. Partager aboutit au morcellement du bien initial. L'échange exprime l'idée d'allers et retours entre plusieurs pôles, à rapprocher de la notion d'équité, de don et de réciprocité. Le vécu des acteurs en la matière est symptomatique d'un malaise sur ces questions. Certains, par exemple, ont l'impression de donner sans contre-partie, s'interrogent sur l'usage qui est fait de l'information qu'ils ont transmise. (Cnet 2001)

D'où l'importance, alors de retravailler la notion de communauté en matière d'information, en l'entendant comme un groupe d'intérêts partagés, pour analyser les conventions, règles implicites et relations de proximité qui structurent sa vie et qui peuvent entrer en conflit avec les comportements individuels. II serait illusoire de penser que la logique de troc et de micro-services d'information mutuellement rendus, basée sur la confiance ou la poursuite d'objectifs communs, viendrait automatiquement s'insérer dans un système collectif, surtout lorsque celui-ci est bâti sur une globalisation d'intérêts hétérogènes.

Quant à sa vie quotidienne et à sa viabilité dans le temps, elles semblent conditionnées par ces questions d'intérêts. L'individu veut avoir autour de lui des réponses à ses problèmes immédiats, ce qui implique que système puisse le prévoir, à la fois pour ce qui le concerne ainsi que pour tous ceux auxquels il s'adresse. La question de l'adéquation, de la convenance, tant des contenus que des modes d'accès, est ainsi posée.

\section{L'exemple des Intranets}

L'Intranet constitue une surface d'observation exemplaire pour illustrer notre approche. Si on l'analyse sur un plan organisationnel, force est de constater que la présentation unifiée cache de multiples configurations, qui signalent l'existence d'un système général (ce qu'il affiche être) et de systèmes locaux. Dans l'absolu, chaque service produit ou donne des informations censées servir à tous. En réalité, il y a presqu'autant d'intranets locaux que de services, quelque peu artificiellement agencés et unifiés grâce à une charte graphique commune. II y a ainsi deux parties, l'une, ouverte, obéissant à une logique de diffusion généralisée telle que la suivent par exemple la communication ou la documentation, et la seconde, qui apparaît comme beaucoup plus ambiguë : certains intranets locaux se construisant autour de leurs intérêts spécifiques, considérant alors leurs informations comme confidentielles. D'autres produisent à la fois des informations valables pour d'autres qu'eux tout en se réservant l'accès à leurs informations propres. L'accès au réseau est alors limité par les mots de passe exigés par chaque service, ce qui restreint alors sérieusement l'espace commun. Cette cohabitation de logiques contradictoires qui contribuent à fermer ou à ouvrir le système, s'établit selon des règles qui sont loin d'être clairement affichées.

Du côté des usages, ce morcellement pose des problèmes aussi bien à l'utilisateur qu'au management. L'utilisateur demande des formes très concrètes d'accessibilité technique et heuristique ainsi que des informations directement adaptées à son cas. Les restrictions d'accès occasionnent de sa part de nouvelles stratégies pour obtenir l'information dont il a besoin pour travailler, ce qui contredit les objectifs de gain de temps. De plus, l'accroissement souvent exponentiel des 
informations publiées sur les sites internes lui pose problème dans la mesure où il lui faut se maintenir en permanence au courant des nouveautés. Cela conduit à accroitre l'activité d'information, ce qui est contradictoire avec les efforts développés pour organiser une disponibilité efficace visant à la réduire.

Le management, lui, cherche à réguler le système en matière de droits et de devoirs, de volumes, de pertinence. On pourrait supposer un niveau central qui garantirait le bien commun et l'égalité de l'accès face à un niveau local protégeant ses intérêts propres. Cela suppose alors une distribution de la légitimité entre ces deux niveaux pour assurer la gestion des droits, la répartition des responsabilités. Nous avons pu constater une grande diversité dans ces modes de régulation, résultant de stratégies de la part d'acteurs que la chose intéresse, allant de pair avec un manque d'arbitrage au niveau central devant des stratégies de surprotection ou au contraire de diffusion excessive de la part des services. Enfin, le manque de critères de référence signale une absence de réflexion sur les contenus et sur leur adéquation avec l'action.

Dans les discours actuels, il semble communément admis que la création d'un espace commun à plusieurs entités entraîne automatiquement échanges et mutualisation. Or le fait est qu'il implique une proximité entre les acteurs concernés et une communauté d'intérêts. L'un des effets du réseau est de contribuer à les identifier et à les structurer en leur donnant les moyens de s'exprimer. Mais cela constitue aussi une forme de privatisation, qui pousse par exemple les services producteurs à s'ériger en propriétaires des informations qu'ils détiennent (et non pas seulement celles qu'ils ont produites), les considérant comme leur propriété et faisant preuve de réticence à les partager. L'accroissement du partage va de pair avec un renforcement des replis.

Quant à la fonction éditoriale, elle est diversement assumée par les webmestres : certains assurent la totalité du travail d'identification des informations, de collecte auprès des différents services, leur sélection et leur organisation en rubriques, la mise en place des modalités d'accès, sans compter la maintenance éditoriale du site et le respect des règles de présentation. Dans d'autres cas, ils n'assurent que la publication du contenu sur le réseau, sans intervenir sur lui. La responsabilité éditoriale revient alors à chaque entité. Alors que l'intranet se déclare être un outil de cohésion et d'identité, les stratégies observées montrent des oscillations entre diffusion et protection, et des réactions de méfiance venant contredire la confiance qu'on aurait pu attendre de personnels appartenant à la même entreprise. II est certain que les restructurations fréquentes et le développement de grands groupes ne favorisent pas une telle perception.

\section{Conclusion}

L'activité d'information, entendue comme étant ce qui se joue autour et avec l'information, tant au plan individuel que collectif, constitue donc un champ d'observation précieux pour réfléchir aux transformations en cours en matière d'information, dorénavant associée à la compétitivité et à l'efficacité. Cependant, le statut de cette activité reste ambigu puisqu'elle est la plupart du temps invisible, voire gênante, aux yeux d'un management qui fixe son regard sur les résultats de l'activité principale ou sur la cohérence structurelle. Quoique nécessaire à l'accomplissement d'une action, elle constitue souvent un investissement lourd en moyens et en compétences, alors même que sa productivité est difficile à mesurer. Aujourd'hui, elle s'élargit, en impliquant les acteurs dans l'alimentation de systèmes collectifs, prenant alors une dimension managériale.

Actuellement, la reconnaissance de cette activité passe par son instrumentalisation qui laisse croire que la rationalisation pourrait être parfaite. Mais affirmer que l'information est devenue un atout dans la guerre économique suppose, en contre-partie, de réfléchir au plus près de l'acteur, à ses méthodes d'information qui constituent sa marge de liberté, à condition d'en posséder les compétences manipulatoires et heuristiques. L'entourer de systèmes et d'outils de tous ordres en espérant qu'il s'en servira se révèle utopique en l'absence de réflexion sur l'accompagnement et les enjeux des nouvelles formes de travail collectif et des objets qui le matérialisent.

Dans des situations où les processus sont clairement balisés, comme pour la production industrielle, la régulation des circuits et des moyens d'information est une tâche lourde mais possible. Dans le cas des fonctions intellectuelles, comme nous le montrent les entretiens menés auprès d'attachés commerciaux, de chargés d'études ou de mission, la rationalisation est plus délicate pour plusieurs raisons. La marge d'autonomie y est plus grande, une part importante est consacrée à la gestion de l'information, que ce soit celle de ses propres dossiers que le recours à ceux des autres. La rédaction 
d'un rapport, la préparation d'une négociation ou d'une mission, nécessitent des informations obtenues en mettant en place des stratégies fort diverses. Chacun maîtrise un secteur dont il assume la responsabilité, dans des relations parfois concurrentielles, et il n'est toujours désireux de le partager.

Le management de l'information constitue l'un des outils accompagnant le mouvement de rationalisation de l'organisation du travail, par le biais d'outils d'information sophistiqués, de procédures de saisie et d'interrogation, d'un formatage de données. Mais sa position est ambiguë, tenant à la situation et à la légitimité de ceux qui l'assument, mais, plus largement au fait que l'information constitue à la fois un facteur d'identité et un facteur d'efficacité, qui joue sur deux registres différents. II faut à la fois gérer la représentation (d'un savoir ou d'une réalité) et la matérialité du document, travailler en même temps sur le sens, l'interprétation, que sur les problèmes très pratiques d'identification, de stockage et d'accès. En outre, ce management doit en permanence intégrer deux démarches pouvant entrer en contradiction: celle de l'acteur, qui est expert d'un domaine et non de la recherche d'information; et celle d'une mise en ordre des moyens d'information qui s'accompagne d'une vigilance aux modes de vie et d'usage de l'information. L'articulation de ces deux aspects suppose d'être attentif aux aspects psychologique, cognitif, social, pédagogique et organisationnel, ce qui complexifie les réflexions.

L'activité d'information semble alors se dérober aux tentatives d'une modélisation totale fondée sur la reproductibilité et une stabilisation des situations. En dehors même de celles-ci, une même personne se comporte différemment selon les moments, l'imprévu ou même l'humeur, ce qui entrave les tentatives parfois très sophistiquées pour personnaliser et construire des profils d'intérêts pertinents. De plus, la diversité même des outils exige de lui des comportements adaptés parfois très différenciés.

De nombreuses questions restent ouvertes, comme celle des limites de l'encadrement de ce qui constitue l'une des marques de l'autonomie de l'acteur, autonomie qui a toujours constitué un défi pour le management, ou celle de la résolution de la contradiction entre l'autonomie et le partage imposé.

Reportant notre regard sur l'acteur, celui-ci apparaît dans une situation ambiguë. Tout d'abord, le fait qu'on commence à ausculter ses manières de vivre avec l'information pourrait s'interpréter comme une reconnaissance du travail intellectuel et organisationnel qu'il effectue depuis sa place, pour luimême et pour les autres. Mais cette reconnaissance est à double tranchant. De nouvelles formes d'imposition électronique font irruption et se rapprochent de son intimité. En lui demandant désormais de formaliser ses gestes et réflexions, ses projets et résultats, voire de penser selon des règles édictées en dehors de lui, constitue une nouvelle pression venant s'ajouter à celles que qu'il connaît aujourd'hui dans son travail.

\section{éléments bibliographiques}

Barbie JM, Kaddouri,(1996) revue éducation permanente, $n^{\circ} 128,1996$, consacré à l'identité.

Bartoli, Le Moigne (1996, Organisation intelligente et système d'information stratégique, Economica Bouillon Jean-Luc (1999), vers une approche commumnicationnelle de la gestion des connaissances, Toulouse III

Cnet, coll, Flexibilisation des organisations productives et du travail, et développement des systèmes d'information multi-média. Rapport final Contrat CNET, mars 2001-Enssib-Gresi, GRIS

Delcambre Pierre (1997), écriture et communications de travail : pratiques d'écriture des éducateurs spécialisés, Lille, éd du septentrion

Dossier : les conventions, Revue Réseaux, $n^{\circ}$ 62, 1993

Doty Philip (2001), Digital privacy : toward a new politics and discursive practices, in annual review or information science and technology, vol 35

Fondin Hubert (2001), pour une épistémologie de la science de l'information, in revue Documentaliste sciences de l'information, $n^{\circ} 2$, juin

Guyot Brigitte (2000), les dynamiques informationnelles, note d'HDR, Grenoble III

Guyot Brigitte (2001), une activité de travail méconnue : l'activité d'information, communication au colloque ICUST, usages et services d'information, Paris, France Telecom, disponible sur : http://www.brigitte-guyot.com Jeanneret Yves (2000), Y a-t-il (vraiment) des technologies de l'information ? Lille, éd du Septentrion 
Latour Bruno (1996), ces réseaux que la raison ignore : laboratoires, bibliothèques, collections, in : le pouvoir des bibliothèques, albin Michel. Disponible sur http://www.ensmp.fr/-latour

Le Coadic Yves (1998), le besoin d'information, éd $A D B S$,

Le Moigne JeanLouis (1997) La théorie du système général, théorie de la modélisation, PUF

Lepine Valérie (2000), les enjeux communicationnels et socio-organisationnels du déploiement de dispositifs de groupware en entreprise, Thèse soutenue à Grenoble.

March James (1987), systèmes d'information et prise de décision : des liens ambigüs, in : décisions et organisations, éd d'organisation

Martin Fabienne (1997), réunions décisionnelles médiatisées par ordinateur en entreprise, Thèse, Lyon II

Revue française de gestion (2000) : immatérielle nouvelle approche de la gestion, $n^{\circ} 130$

Réseaux (1993), dossier sur les conventions, $n^{\circ} 62$

Reynaud Jean-Daniel (1989), les règles du jeu : action collective et régulation sociale Armand Colin

Simon Herbert (1955) A behavorial model of rational choice, quaterly Journal of ectoproctes $n^{\circ} 69$

Terssac Gilbert de (1992), Autonomie dans le travail, PUF

Vacher Béatrice (1997), la gestion de l'information dans l'entreprise, ADBS,

Wright (1951), les cols blancs, Seuil

Zarifian Philippe (1997), travail et communication, PUF

\footnotetext{
${ }^{1}$ Nous pensons aussi à des thèses plus anciennes, comme celle de Sylvie Craipeau (techniques de l'organisation et organisation de la technique, 1980), ou de Robert Panico (les informaticiens confrontés aux nouvelles technologies d'information, Grenoble, 1994)

${ }^{2}$ voir les travaux en cours du programme régional ISDN sur le document numérique, en particulier un axe sur la structuration et l'enrichissement du document, http://
} 